\title{
artigo
}

Fraga, J.M.; Rodrigues da Silva, A.; Oliveira, A.R.S.; Barbosa, A.S.; Girão, C.M.; Studart, R.M.

Fatores que influenciam o tempo de internação no transplante renal

\section{Fatores que influenciam o tempo de internação no transplante renal}

\author{
Factors that influence the time of hospitalization in kidney transplantation \\ Factores que influyen en el tiempo de hospitalización en el trasplante de riñón
}

\begin{abstract}
RESUMO
Objetivo: Identificar os elementos que interferem no tempo de internação do paciente transplantado renal. Método: Trata-se de um estudo transversal e retrospectivo realizado em um hospital de Fortaleza. Foram analisados 236 prontuários de pacientes transplantados, entre 2017 e 2019. Utilizou-se uma planilha eletrônica para organização dos dados, que posteriormente foram analisados no programa Statistical Package for the Social Sciences. Resultados: Constatou-se que a maioria possuía a doença de base de etiologia desconhecida, diabetes e hipertensão. Dos 236 pacientes, 40,3\% tiveram função imediata do enxerto, 80,9\% não apresentaram nenhum foco de infecção, com média de 11 dias de internação. Aqueles que apresentaram creatinina normal passaram menos dias internado. Conclusão: A creatinina, a função tardia do enxerto e infecção foram fatores diretamente relacionados com o tempo de internação hospitalar, sendo essencial que os profissionais busquem estratégias para evitar e/ou minimizar as complicações no pós-transplante.
\end{abstract}

DESCRITORES: Hospitalização; Tempo de Internação; Transplante Renal;

\section{ABSTRACT}

Objective: Identify the elements that interfere with the length of hospital stay for kidney transplant patients. Method: This is a cross-sectional and retrospective study conducted at a hospital in Fortaleza. 236 medical records of transplant patients were analyzed between 2017 and 2019. An electronic spreadsheet was used to organize the data, which were later analyzed using the Statistical Package for the Social Sciences program. Results: It was found that the majority had the underlying disease of unknown etiology, diabetes and hypertension. Of the 236 patients, $40.3 \%$ had immediate graft function, $80.9 \%$ did not have any infection, with an average of 11 days of hospitalization. Those who had normal creatinine spent less days in the hospital. Conclusion: Creatinine, late graft function and infection were factors directly related to the length of hospital stay, and it is essential that professionals seek strategies to avoid and / or minimize complications in the post-transplant period.

DESCRIPTORS: Hospitalization; Hospitalization time; Kidney Transplantation;

\section{RESUMEN}

Objetivo: Identificar los elementos que interfieren con la duración de la estancia hospitalaria de los pacientes con trasplante de riñón. Método: Se trata de un estudio transversal y retrospectivo realizado en un hospital de Fortaleza. Se analizaron 236 historias clínicas de pacientes trasplantados entre 2017 y 2019. Se utilizó una hoja de cálculo electrónica para organizar los datos, que luego se analizaron mediante el Paquete Estadístico del Programa de Ciencias Sociales. Resultados: Se encontró que la mayoría tenía la enfermedad de base de etiología desconocida, diabetes e hipertensión. De los 236 pacientes, el 40,3\% tenía función inmediata del injerto, el 80,9\% no presentaba infección, con un promedio de 11 días de hospitalización. Aquellos que tenían creatinina normal pasaron menos días en el hospital. Conclusión: La creatinina, la función tardía del injerto y la infección fueron factores directamente relacionados con la duración de la estancia hospitalaria, siendo fundamental que los profesionales busquen estrategias para evitar y / o minimizar las complicaciones en el postrasplante.

DESCRIPTORES: Hospitalización; Tiempo de hospitalización; Trasplante de riñón;

RECEBIDO EM: 28/04/2021 APROVADO EM: 18/05/2021

\section{Jamila Moura Fraga}

Enfermeira, Especialista na Residência em Transplante de Órgãos e Tecidos - Hospital Geral de Fortaleza - HGF, Fortaleza, Ceará. ORCID: 0000-0003-4775-9885 
Alan Rodrigues da Silva

Farmacêutico, Mestre em Transplante de Órgãos e Tecidos - UECE, Hospital Geral de Fortaleza - HGF, Fortaleza, Ceará. ORCID: 0000-0002-9633-363X

\section{Antonia Rozângela Souza de Oliveira}

Enfermeira. Residente em Transplante de Órgãos e Tecidos - Hospital Geral de Fortaleza - HGF, Fortaleza, Ceará. ORCID: 0000-0002-8803-6213

\section{Aglauvanir Soares Barbosa}

Enfermeira, Mestre pela Universidade da Integração Internacional da Lusofonia Afro-Brasileira. ORCID: 0000-0003-4909-563X

\section{Celi Melo Girão}

Enfermeira, Mestre em Saúde da Comunidade, especialista em administração hospitalar, nefrologia, gestão da clínica e saúde da comunidade. ORCID: 0000-0001-8694-742X

\section{Rita Monica Borges Studart}

Enfermeira, Doutora em enfermagem pela UFC. Professora adjunto da Universidade de Fortaleza. ORCID: 0000-0002-5862-5244

\section{INTRODUÇÃO}

0 Transplante é uma opção terapêutica confiável e com grande eficácia que visa proporcionar a reabilitação e aumento da qualidade de vida em pacientes com falência algum órgão ou tecido. Dentre os diversos tipos de transplantes realizados encontra-se o transplante de rim ${ }^{1}$.

O Transplante renal $(\mathrm{TxR})$ está indicado para pacientes com Taxa de Filtração Glomerular (TFG) menor que $15 \mathrm{ml} /$ min, estando ou não em tratamento dialítico. O mesmo oferece maior sobrevida, melhor qualidade de vida e, por vezes, é o único recurso para manutenção da vida do indivíduo ${ }^{2}$.

Este é o tratamento de escolha para muitos pacientes que sofrem de doença renal em estágio terminal (DRET) e possui poucas contraindicações. Sabe-se que a vantagem de sobrevivência do TxR em comparação com a hemodiálise (HD) para pacientes com DRET está bem estabelecido, principalmente devido a qualidade de vida pós transplante e os custos diferentes das duas terapias substitutivas ${ }^{3,4}$.

$\mathrm{O}$ TxR é considerado um procedimento cirúrgico simples e após o implante são necessárias algumas intervenções essenciais para manutenção do enxerto, tais como o uso de medicamentos imunossupressores, cuidados com higiene e prevenção de infec- ção, além do acompanhamento pós TxR, que se inicia logo após a chegada do paciente na Unidade de Terapia Intensiva (UTI) e se estende por toda a vidá

Ressalta-se que, mesmo o TxR sendo uma alternativa com inúmeros benefícios, ela pode culminar em complicações relevantes, dentre as quais podemos citar: função retardada do enxerto (FRE), hipovolemia, necrose tubular aguda (NTA), rejeição hiperaguda e aguda, nefrotoxidade por inibidores da calcineurina da ciclosporina e oclusão vascular ${ }^{5}$.

Outra complicação comum é a infecção pós-transplante, que está relacionada à modulação do sistema imunológico do receptor, sendo considerada a segunda causa de morte entre os pacientes portadores de insuficiência renal crônica e responsável por mais de 50\% das complicações no pós-operatório ${ }^{6}$. Dessa forma, o período de permanência hospitalar e a qualidade do cuidado pós-operatório são considerados fatores de risco para infecções?

Entende-se que todas essas complicações resulta em um processo prolongado na recuperação da função do enxerto, consequentemente contribui com o aumento do tempo de internação, o que poderá também colaborar para ocorrência de infecção, culminando em pior sobrevida do enxerto a longo prazo e condições de saúde do paciente.

A redução do tempo de internação hospitalar (TIH) é crucial para a diminuição dos riscos inerentes a internação prolongada. A hospitalização prolongada é um dos problemas que mais prejudica o sistema de saúde. Visto que, o paciente está sujeito a riscos desnecessários, como alterações psicológicas, risco de infecção, alteração do sono, dentre outros distúrbios. Além disso, a internação prolongada influencia e aumenta os custos financeiros custeados pelo hospital ${ }^{9}$.

Com relação ao TxR, a média de permanência hospitalar corresponde a 11 dias de internação. Considera-se hospitalização prolongada pacientes que permaneceram mais de 12 dias de internação hospitalar (DIH) pós-transplante ${ }^{10}$. Deste modo, considerando a relevância do TxR, o estudo teve como objetivo identificar os elementos que interferem no tempo de internação do paciente transplantado renal.

\section{MÉTODOLOGIA}

Trata-se de um estudo com delineamento transversal e retrospectivo. A amostra foi constituída por pacientes submetidos ao transplante renal com doador falecido em um hospital de Fortaleza referência em transplante de órgãos e tecidos, no período de junho de 2017 a junho de 2019, na faixa etária de 18 a 78 anos de idade.

A coleta dos dados foi realizada de janeiro a maio de 2020. Foram avaliados 236 prontuários de pacientes transplantados re- 
nais que realizaram o transplante na referida instituição. Foram selecionados pacientes que atendiam aos critérios de inclusão, transplante renal com doador falecido e critério de exclusão, transplante duplo de pâncreas-rim, prontuário com informações insuficientes para realização da pesquisa, receptores com idade inferior a 18 anos e óbito após o transplante.

Foram avaliados os dados sociodemográficos, comorbidades, doença de base que levou a falência renal, tempo em diálise, creatinina antes do transplante, creatinina do dia da alta hospitalar, função tardia do enxerto (FTE), número de HD pós transplante, função do enxerto, rejeição, infecção e o TIH (do dia transplante até o dia da alta hospitalar).

A entrada dos dados quantitativos foi realizada usando-se a planilha eletrônica Excel 2003 for Windows, sendo estes dados posteriormente submetidos à análise estatística por meio do programa Statistical Package for the Social Sciences (SPSS versão 23.0). Estatísticas descritivas foram realizadas para as variáveis qualitativas por meio de frequências absolutas e relativas. Para as variáveis quantitativas contínuas e discretas foram calculadas a média, desvio-padrão (DP), mediana, mínimo e máximo.
Tabela 1 - IMC, doença de base, comorbidades e tempo em diálise dos pacientes antes do transplante $(n=236)$

\section{VARIÁVEIS}

N

IMC

Normal (18-24)

Baixo peso (<18)

Sobrepeso $(25-29,9)$

Obeso (>30)

Doença de base

DM

HAS

Glomerulopatias

Doença Policística Renal

Doença urológica/Bexiga neurogênica

Etiologia desconhecida

Outros

Comorbidades

Sem comorbidades

DM

HAS

$\mathrm{DM}+\mathrm{HAS}$

Problemas vasculares

Outras

Tempo em diálise (pré TxR)

Preempitivo

Até 12 meses

De 12 a 36 meses

$>36$ meses

Fonte: Dados da pesquisa, 2021. n - número total; \% porcentagem; DP- Desvio Padrão; IMC- Indice de Massa CorporalHAS- Hipertensão Arterial Sistemica; DM- Diabetes Mellitus;
A normalidade das variáveis foi verificada pelo teste Kolmogorov-Smirnov.

Para análise dos fatores intervenientes nos dias de internação entre os participantes da pesquisa, buscou-se estratificar o tempo de internação em normal (até 11 dias) e prolongado (acima de 11 dias). A associação entre as variáveis valor de creatinina, função do enxerto, tempo de HD (antes do transplante), número de HD pós-transplante, comorbidades, infecções e o desfecho dos dias de internação (normal ou prolongado) foram avaliadas pelo teste do qui-quadrado de Pearson, sendo considerado estatisticamente significativo o valor de $\mathrm{p}<0,05$, a força dessa associação pelo cálculo das razões de chances (Odds Ratio - OR) e regressão logística pelo método backward para ajuste do modelo. Para entrada das variáveis no modelo, foi considerado o $\mathrm{p}<0,20$ e para a sua permanência o $\mathrm{p}<0,05$.

Este estudo foi aprovado pelo Comitê de Ética em Pesquisa do Hospital Geral de Fortaleza, sob o Parecer 3.348.699.

\section{RESULTADOS}

As Variáveis contínuas foram apresentadas como média, desvio padrão e variância. As variáveis categóricas foram apresentadas como frequência e porcentagem. A amostra foi composta por 236 pacientes transplantados renais, com idade média de 46,8 \pm 13,8 anos, 144 (61\%) eram do sexo masculino, pardos (202; $85,6 \%$ ) e de procedência do interior do estado (114;48,3\%). Em relação a situação conjugal, $143(60,6 \%)$ eram casados, $71(30,1 \%)$ solteiros, e os demais divorciados, com união estável ou viúvos $(12,4,6$, respectivamente). Com relação a escolaridade, a maioria havia concluído o ensino médio (104; 44,1\%), 84(35,3\%) cursaram até o ensino fundamental, 31 (13,1\%) possuem ensino superior e os demais não eram alfabetizados $(17 ; 7,2 \%)$.

A doença de base que mais levou o paciente a falência renal, era de etiologia desconhecida (37,7\%), seguido de Diabetes Mellitus (17,8\%) e glomerulopatias (16,9\%). Já em relação as comorbidades associadas a doença de base, $60,6 \%$ dos pacientes com falência renal apresentavam HAS. Além disso, cerca de 20,3\% possuíam HAS e DM concomitante. 
A creatinina média dos receptores antes do TxR era 7,8 $\pm 3,6 \mathrm{mg} / \mathrm{dl}$, apresentando uma grande variância de $13,1 \mathrm{mg} /$ dl. Já com relação a creatinina no dia da alta hospitalar, a média foi de $3,1 \pm 2,3$ $\mathrm{mg} / \mathrm{dl}$, com variância menor, $5,7 \mathrm{mg} / \mathrm{dl}$.

Dos 236 pacientes, 95 (40,3\%) tiveram função imediata do enxerto (FIE). No entanto, 7 (3\%) pacientes tiveram rejeição do enxerto, com média de $44 \mathrm{DIH}$, variando de 15 a 94 dias. A maioria dos participantes não presentou FTE (152;

Tabela 2. Fatores intervenientes no tempo de internação (normal ou prolongado)

VARIÁVEIS

Comorbidades

Sim

Não

Função do enxerto

FTE

Sim

Função lenta

$\operatorname{Sim}$

Não

Imediata

Sim

Não

Perda

Sim

Não

Creatinina

Alta

Normal

Rejeição

Sim

Não

HD antes do TxR

Até 3,2 meses

Acima de 3,2 meses

HD pós-transplante

$$
\text { Sim }
$$

Não

Infecção

$\operatorname{Sim}$

Não

\section{DIAS DE INTERNAÇÃO}

\section{NORMAL}

N

$\%$

PROLONGADO

$\%$

110

20

53,1

69,0

97

46,9

31,0

$0,109 a$

$0,51(0,22-1,17)$

1

$0,001 a$

81,5

44,9

$115 \quad 74,2 \quad 40$

$0,624 a$

42,1

45,8

$97 \quad 54,2 \quad 82$

81

85,3

14

14,7

65,2

66,7

44,6

0,001a

50,0

12,5

28

87,5

04

07

100

43,2

0,113a

0,001b

$130 \quad 56,8 \quad 99$

76

59,8

51

40,2

50,5

$54 \quad 49,5 \quad 55$

$17 \quad 20,7$

65

79,3

113

73,4

41

26,6

0,001a

0,001a

$10,53(5,54-20,03)$

1

$2,31(1,99-2,68)$

1

$1,51(0,90-2,54)$

1

Legenda: a = Qui-Quadrado de Pearson; $b$ = Razão de Verossimilhança; $c$ = Odds ratio (intervalo de confiança de 95\%); TxR= Transplante Renal. Fonte: Elaboração própria.
64,4\%), 2 destes tiveram perda do enxerto em decorrência de complicações no pós operatório e $82(34,7 \%)$ pacientes tiveram FTE, com média de $2 \pm 4,2 \mathrm{HD}$ pós TxR, com grande variância de 18,3.

A infecção está dentre os fatores que contribuem para o aumento no tempo de internação hospitalar. Cerca de 190 $(80,9 \%)$ pacientes não apresentaram nenhum foco de infecção após TxR. Os demais pacientes tiveram infecção de foco urinário $(23 ; 9,8 \%)$, infecção de ferida operatória $(8 ; 3,4 \%)$, infecção respiratória $(3 ; 1,3 \%)$ e outros focos infecciosos (11; 4,7\%).

Os dias de internação dos pacientes participantes desta pesquisa variaram entre três a 139 dias, com mediana de 11 dias. Assim, observou-se que a maioria dos pacientes $(130 ; 55,1 \%)$ ficaram internados por um período normal e 106 (44,9\%), por um período prolongado.

No que concerne às associaçóes entre as variáveis e o desfecho dias de internação (normal e prolongado) (Tabela 1), observou-se associação significativa entre FTE $(\mathrm{p}<0,001)$ e FIE $(\mathrm{p}<0,001)$, valor de creatinina $(p<0,001)$, rejeição $(p<0,001)$ e infecção $(p<0,001)$ com os dias de internação. Apenas as variáveis de função lenta e perda (função do enxerto) não entraram no modelo de regressão.

Conforme mostra a Tabela 2, permaneceu no modelo final da regressão apenas o valor de creatinina $(\mathrm{p}=0,012$; $\mathrm{OR}=$ $0,230[0,070-0,810])$, infecção $(p<0,001$; $\mathrm{OR}=15,568[5,613-43,178]) \quad$ e $\quad$ FIE $(\mathrm{p}<0,001 ; \quad O R=5,183[2,136-12,576]) \quad \mathrm{e}$ FTE $(p=0,001 ; O F=0,172[0,076-0,391])$.

$\mathrm{O}$ valor de creatinina revelou-se como fator protetor nos participantes desta pesquisa, ou seja, aqueles que apresentaram creatinina dentro dos valores normais, passaram menos dias internados. Os pacientes que tiveram FTE não apresentaram maior tempo de internação, mesmo sendo um dos fatores que podem aumentar os dias de hospitalização. Destes que tiveram FTE, 25,2\% dialisaram entre $1 \mathrm{e}$ 6 vezes após TxR. Os pacientes que apresentaram alguma infecção e FIE tiveram 15,568 e 5,183 vezes mais chances, respec- 
tivamente, de permanecerem internados por tempo prolongado.

\section{DISCUSSÃO}

No geral, a amostra estudada foi predominantemente do sexo masculino, com média de idade de 46,8 $\pm 13,8$ anos, a maioria com ensino médio completo, corroborando com os dados apresentados na pesquisa realizada por Magalhães e colaboradores (2019) e Dantas e colaboradores (2020) que também apresentou predominância do sexo masculino, com a média de idade de 42 anos, apresentando um desvio padrão maior entre $\pm 14-17,5$ anos.

A doença de base que mais levou os pacientes a doença renal crônica foram de etiologia desconhecida, seguido de DM, glomerulopatias e HAS, respectivamente. O que corrobora com os dados encontrados no estudo de Sandes-Freitas e colaboradores (2015) e Baid-Agrawal e colaboradores (2016), no qual DM, HAS e glomerulopatias também foram as principais causas de lesão renal que levaram o paciente ao transplante ${ }^{11,13}$.O que leva a uma preocupação relacionada ao desenvolvimento de FTE, já que os receptores que tiveram como doença de base HAS e DM tem mais probabilidade de desenvolver essa complicação. Da mesma forma, a DM foi um fator de risco para perda do enxerto renal em 1 ano, assim como um fator de risco associado a mortalidade ${ }^{14,15}$.

Apenas 29 pacientes não apresentavam nenhuma comorbidade além da doença do base. A maioria dos receptores (143, $60,6 \%$ ) possuíam somente HAS como comorbidade, e cerca de $20,3 \%$ apresentavam DM associada a HAS. Já com relação ao tempo em diálise, apenas um pequeno grupo não dialisava antes do transplante $(15,6,4 \%)$. No entanto, a maioria dos pacientes $(117,49,6 \%)$ passaram um longo período em diálise, mais de 36 meses. O mesmo dado também foi encontrado em outra pesquisa, em que o tempo em dialise antes do TxR é mais de 60 meses $^{2,16}$. Os demais receptores que tiveram esse tratamento por um período de 12 a 36 meses (89; 37,7\%). Apenas uma pequena minoria conseguiu transplantar antes de 12 meses de diálise. Por conseguinte, outros estudos apontam que os pacientes que tiveram o maior tempo médio em diálise, apresentaram FTE e maior risco de óbito após $\mathrm{TxR}^{12,17}$.

A amostra do estudo em questão evidenciou que 95 pacientes tiveram FIE e não apresentaram FTE $(152 ; 64,4 \%)$. A literatura reforça que, pacientes com FIE têm a evolução significativamente melhor durante o primeiro ano de transplante e maior sobrevida do enxerto do que pacientes com FTE ${ }^{18}$.

Uma análise multivariada mostrou que o retransplante, a idade do doador, a creatinina sérica final, o tempo de isquemia fria e regime imunossupressor foram as variáveis independentemente associadas à FTE. Nesse contexto, vale ressaltar que ainda há um elevado número de receptores apresentando FTE $(82 ; 34,7 \%)$, o que pode vir a desencadear uma rejeição aguda. Isso se torna ainda mais relevante quando ocorre um aumento no TIH devido a FTE ${ }^{19}$.. Segundo a pesquisa de Sandes-Freitas e colaboradores (2015), a incidência de rejeição aguda em 6 meses foi 3 vezes maior em pacientes com FTE em comparação com pacientes sem FTE. $\mathrm{O}$ mesmo estudo apontou que a maioria dos episódios de rejeição aguda ocorreu durante o período FTE ${ }^{12,20}$. Desta forma, observou-se que houve o aumento no risco de rejeição e menor sobrevida do enxerto naqueles pacientes que tiveram FTE, o que também foi observado no estudo de Helfer e colaboradores (2019) ${ }^{21}$. Os dados apresentados reforçam o impacto negativo que a FTE tem nos desfechos do transplante e confirmam o risco de sua ocorrência ${ }^{22}$.

A infecção está dentre os fatores que contribuem para o aumento no tempo de internação hospitalar ${ }^{19}$. Cerca de 190 $(80,9 \%)$ pacientes não apresentaram nenhum foco de infecção após TxR. Os demais pacientes tiveram infecção de foco urinário $(23 ; 9,8 \%)$, infecção de ferida operatória $(8 ; 3,4 \%)$, infecção respiratória (3; $1,3 \%)$ e outros focos infecciosos (11; 4,7\%). Sabe-se que a infecção está intimamen- te relacionada com o maior TIH23. Analisando os resultados obtidos, observou-se que 44 pacientes que tiveram infecção, apresentaram o tempo de internação prolongado, e apenas 7 apresentaram infecção mas não excederam os $11 \mathrm{DIH}$. Dados do estudo de Corrêa e colaboradores (2013) apontam que ocorreu associação estatisticamente significativa entre rejeição e infecção. $\mathrm{O}$ mesmo estudo aponta que os pacientes que desenvolveram infecção, $44,1 \%$ apresentaram rejeição e $28,8 \%$ não apresentaram rejeição. Já com relação aos pacientes sem infecção, $71,2 \%$ não tiveram rejeição e 55,9\% sim. Os dados apresentados reforçam a importância de diminuir os DIH, reduzindo assim o risco de infecção e complicações após o TxR.

A mediana dos DIH foi de 11 dias, assim, observou-se que a maioria dos pacientes $(130 ; 55,1 \%)$ ficaram internados por um período normal e 106 (44,9\%), por um período prolongado. Segundo Santos e colaboradores (2016), uma maior ocorrência de rejeição está relacionada ao maior tempo de internação. Outras características do receptor também estão associadas ao tempo de permanência mais longo, são elas o aumento da idade, o tempo de HD, retransplantes, DM e entre outros ${ }^{23,5}$.

O TIH prolongado está associado a maiores taxas de rejeição e infecção ${ }^{8}$. O que leva a analisar todos os fatores que influenciam o aumento no tempo de internação do paciente, aumentando assim as chances de complicação no pós TxR. No presente estudo o valor de creatinina revelou-se como fator protetor nos participantes desta pesquisa, ou seja, aqueles que apresentaram declínio maior no valor da creatinina para dentro dos valores normais, passaram menos dias internados. $\mathrm{O}$ que diverge da literatura são os pacientes que tiveram FTE mas não apresentaram maior tempo de internação, mesmo sendo um dos fatores que podem aumentar os dias de hospitalização.

\section{CONCLUSÃO}

É essencial que os profissionais da saúde saibam os fatores que influen- 
ciam na evolução, no tempo de internação do paciente e busquem estratégias para evitar e/ou minimizar as complicações no pós transplante. Os dados do estudo revelam que grande parte dos resultados obtidos corroboram com as pesquisas já realizadas e publicadas. Em suma, a função imediata do enxerto, função tardia do enxerto, infecção e creatinina sérica do receptor no pós transplante renal estão diretamente relacionadas com o tempo de internação hospitalar. Estes foram os elementos identificados como fatores intervenientes no tempo de internação hospitalar após o transplante renal.

Ainda assim, alguns fatores do aumento do tempo de internação hospitalar podem estar relacionados ao perfil dos receptores, às condições ambientais, às práticas assistenciais, às técnicas cirúrgicas e principalmente ao doador. Apesar dos resultados apresentados, ainda se faz necessário pesquisas mais robustas e qualificadas para avaliar os resultados com mais variáveis, para assim acrescer os estudos já realizados.

\section{REFERÊNCIAS}

1. Clementino J, Campos R, Gonzaga H, Neves H, Machado M, Carvalho P. Atuação do enfermeiro na organização para procura de órgãos: estudo de caso único institucional. Saúde Coletiva (Barueri). 2020;10(58):3959-70.

2. Magalhães ALP, Campos TS, Lima VGK, Lins SMSB, Souza E. Adesão à terapia imunossupressora de transplantados renais de um hospital universitário. Saúde Coletiva (Barueri). 2019;9(51):1892-8.

3. Silva, SB, Caulliraux, HM, Araújo, CAS, \& Rocha, E. Uma comparação dos custos do transplante renal em relação às diálises no Brasil. Cadernos de Saúde Pública. 2016: 32: e00013515.

4. Santos LF, Prado, BDC, Castro, FPDS, Brito, RF, Maciel, SC, \& Avelar, TC. Qualidade de Vida em Transplantados Renais. Psico-usf. 2018: 23(1): 163-172.

5. Carolline A, Torres O, Helena B, Lembi BK, Isidoro S, Fernandes FP, et al. Complicações pós-operatórias em pacientes submetidos a transplante renal: uma revisão narrativa. Rev Eletrônica Acervo Saúde. 2021;13(4):1-7.

6. Starck E, Mittelmann TH, Lovatto MVP, Nakalski LR, Abate DTRS. Complicações infecciosas no primeiro ano pós-transplante renal. Brazilian J Dev. 2020;6(6):36663-76.

7.Maia N, Parada B, Dinis P. Tempo de Isquemia Fria e a Incidência de Complicações Infeciosas após o Transplante Renal. Fac Med da Univ Coimbra. 2017;3(17):1-22.

8. Santos W, Rocha F, Ribeiro I, Coqueiro J. Atuação do enfermeiro nas complicações decorrentes do transplante renal: uma revisão de literatura. Rev Uningá Rev. 2016;25(1):136-42.

9. Silva RP, Pinto PIP, Alencar, AMC. Efeitos da hospitalização prolongada: o impacto da internação na vida paciente e seus cuidadores. Saúde (Santa Maria). 2018: 3(44).

10. Yazawa M, Cseprekal O, Helmick RA, Talwar M, Balaraman V, Podila PS, Molnar MZ. Association between longer hospitalization and development of de novo donor specific antibodies in simultaneous liver-kidney transplant recipients. Renal failure. 2020: 42(1): 40-47.

11. Dantas GC, Galão JA, Pompeo LL, Titenis LV, de Souza FL, Uscocovish VSM. Comparação Da Evolução Pós-Operatória Precoce Em Pacientes Submetidos A Transplante Renal Com Rins De Doadores Vivos E Falecidos, Na Cidade De Cascavel-Pr. Fag Journal Of Health (Fjh). 2020: 2(2), 172-179.

12. Sandes-Freitas TV, Felipe CR, Aguiar WF, Cristelli MP, Tedes-
co-Silva $\mathrm{H}$, Medina-Pestana JO. Prolonged delayed graft function is associated with inferior patient and kidney allograft survivals. PLoS One. 2015: 10(12), e0144188.

13. Baid-Agrawal S, Martus P, Feldman H, Kramer H. Weekend versus weekday transplant surgery and outcomes after kidney transplantation in the USA: a retrospective national database analysis. BMJ Open. 2016: 6: e010482.

14. Ruppel P, Felipe CR, Medina-Pestana JO, Hiramoto LL, Viana $L$, Ferreira $A$, et al. The influence of clinical, environmental, and socioeconomic factors on five-year patient survival after kidney transplantation. J Bras Nefrol. 2018;40(2):151-61.

15. Ecker R, Beltrame V, Dallacosta F. Mortalidade Pós-Transplante Renal. Rev Interdiscip Estud em Saúde. 2015;9(2):253-60.

16. Mendonça AEO, Torres GDV, Salvetti MDG, Alchieri JC, Costa IKF. Mudanças na qualidade de vida após transplante renal e fatores relacionados. Acta Paulista de enfermagem. 2014: 27(3), 287-292.

17. Riella L V. Entendendo as causas da mortalidade pós-transplante - indo além do que se percebe à primeira vista. J Bras Nefrol. 2018;40(2):102-4.

18. Rodrigo E, Ruiz JC, Piñera C, Fernández Fresnedo G, Escallada $\mathrm{R}$, Palomar $\mathrm{R}$, Arias $\mathrm{M}$. Creatinine reduction ratio on post transplant day two as criterion in defining delayed graft function. American Journal of Transplantation. 2004: 4(7), 1163-1169.

19. Tavares M, Junior $H$, Pestana J. Readmissão Hospitalar Precoce no transplante renal: artigo de revisão. J Bras Nefrol. 2020;42(2):231-7.

20. Gómez-Sánchez M, Gómez-Ziga A, Carcencia-Barajas C, Ortiz-Luis S. Complicaciones de pacientes con trasplante renal en las primeras 48 horas en un hospital de tercer nivel de atención. Rev enferm Inst Mex Seguro Soc. 2019;27(3):154-62.

21. Helfer MS, Pompeo J de C, Costa ORS, Vicari AR, Ribeiro AR, Manfro RC. Efeitos de longo prazo da duração da função tardia do enxerto sobre a função e sobrevida de transplantes renais com doadores falecidos. J Bras Nefrol. 2019;41(2):231-41.

22. Sandes-Freitas TV de. A problemática da função tardia do enxerto renal no Brasil. J Bras Nefrol. 2019;41(2):166-7.

23. Corrêa APA, Brahm MMT, Teixeira CDC, Ferreira SAL, Manfro RC, Lucena ADF, et all. Complicações durante a internação de receptores de transplante renal. Revista Gaúcha de Enfermagem, .2013: 34(3), 46-54. 